\title{
Prognostic Ability of Simplified Pulmonary Embolism Severity Index (PESI) Score in Real World: A Brief Report
}

\section{Luca Masotti $^{1 \text {, 7, 25, * , Grazia Panigada }}{ }^{2}$, Giancarlo Landini ${ }^{1}$, Filippo Pieralli ${ }^{3}$, Francesco Corradi ${ }^{3}$, Salvatore Lenti ${ }^{4}$, Rino Migliacci ${ }^{5}$, Carlo Nozzoli $^{3}$, Maddalena Grazzini ${ }^{3}$, Lucia Ciucciarelli ${ }^{3}$, Alessandro Morettini ${ }^{3}$, Sara Bucherelli ${ }^{3}$, Alessandra Petrioli ${ }^{3}$, Carlotta Casati ${ }^{3}$, Mario Felici ${ }^{4}$, Luciano Ralli ${ }^{4}$, Stefano Arrigucci ${ }^{4}$, Laila Teghini ${ }^{2}$, Giovanni Antonio Porciello ${ }^{5}$, Stefano Spolveri ${ }^{6}$, Daniele Baldoni $^{6}$, Anna Frullini ${ }^{6}$, Barbara Cimolato ${ }^{1}$, Gianni Lorenzini ${ }^{7}$, Alessandro Pampana ${ }^{7}$, Guidantonio Rinaldi ${ }^{8}$, Maria Chiara Bertieri ${ }^{8}$, Raffaele Laureano ${ }^{9}$, Stefano Tatini ${ }^{9}$, Alberto Fortini $^{10}$, Chiara Angotti ${ }^{10}$, Valerio Verdiani ${ }^{11}$, Anna Maria Romagnoli ${ }^{11}$, Irene Cascinelli ${ }^{11}$, Alberto Camaiti $^{12}$, Nicola Mumoli ${ }^{12}$, Marco Cei ${ }^{12}$, Stefano Giuntoli ${ }^{12}$, Massimo Alessandri ${ }^{13}$, Alessandro De Palma ${ }^{13}$, Maurizio Manini ${ }^{14}$, Veronica De Crescenzo ${ }^{14}$, Michele Piacentini ${ }^{15}$, Carlo Passaglia ${ }^{16}$, Giancarlo Tintori ${ }^{16}$, Carlo Palermo ${ }^{17}$, Alba Dainelli ${ }^{17}$, Roberto Andreini ${ }^{18}$, Giuseppa Levantino $^{18}$, Plinio Fabiani ${ }^{19}$, Lucia Raimondi ${ }^{19}$, Massimo Di Natale ${ }^{20}$, Filippo Risaliti ${ }^{20}$, Rossella Nassi ${ }^{21}$, Roberta Mastriforti ${ }^{21}$, Roberto Cappelli ${ }^{22}$, Michele Voglino ${ }^{22}$, Paola Lambelet ${ }^{23}$, Stefano Fascetti ${ }^{23}$, Adriano Cioppi ${ }^{23}$, Valentina Carli $^{23}$, Alessandro Tafi ${ }^{24}$, Simone Meini ${ }^{24}$, Emilio Santoro ${ }^{26}$, Claudia Rosi ${ }^{26}$}

\footnotetext{
${ }^{1}$ Internal Medicine, Santa Maria Nuova Hospital, Florence, Italy

${ }^{2}$ Internal Medicine, Pescia Hospital, Pistoia, Italy

${ }^{3}$ Internal Medicine, Careggi Hospital, Florence, Italy

${ }^{4}$ Internal Medicine, Arezzo Hospital, Arezzo, Italy

${ }^{5}$ Internal Medicine, Cortona Hospital, Arezzo, Italy

${ }^{6}$ Internal Medicine, Borgo San Lorenzo Hospital, Florence, Italy

${ }^{7}$ Internal Medicine, Cecina Hospital, Livorno, Italy

${ }^{8}$ Internal Medicine, Barga Hospital, Lucca, Italy

${ }^{9}$ Internal Medicine, Santa Maria Annunziata Hospital, Florence, Italy

${ }^{10}$ Internal Medicine, San Giovanni di Dio Hospital, Florence, Italy

${ }^{11}$ Internal Medicine, Grosseto Hospital, Grosseto, Italy

${ }^{12}$ Internal Medicine, Livorno Hospital, Livorno, Italy

${ }^{13}$ Internal Medicine, Massa Marittima Hospital, Grosseto, Italy

${ }^{14}$ Internal Medicine, Orbetello Hospital, Grosseto, Italy

${ }^{15}$ Internal Medicine, Piombino Hospital, Livorno, Italy

${ }^{16}$ Internal Medicine, Cisanello Hospital, Pisa, Italy

${ }^{17}$ Internal Medicine, Campostaggia Hospital, Siena, Italy

${ }^{18}$ Internal Medicine, Pontedera Hospital, Florence, Italy

${ }^{19}$ Internal Medicine, Portoferraio Hospital, Livorno, Italy

${ }^{20}$ Internal Medicine, Prato Hospital, Prato, Italy

${ }^{21}$ Internal Medicine, San Sepolcro Hospital, Arezzo, Italy

${ }^{22}$ Internal Medicine, Le Scotte Hospital, Siena, Italy

${ }^{23}$ Internal Medicine, Viareggio Hospital, Viareggio, Italy

${ }^{24}$ Internal Medicine, Volterra Hospital, Volterra, Italy

${ }^{25}$ Internal Medicine, Santa Maria Nuova Hospital, Florence, Italy

${ }^{26}$ Internal Medicine, Bibbiena Hospital, Bibbiena, Italy
}

Email address:

luca.masotti@tin.it (L. Masotti),g.panigada@usl3.toscana.it (G. Panigada), filpiero@tin.it (F. Pieralli), lentisa@libero.it (S. Lenti)

To cite this article:

Luca Masotti, Grazia Panigada, Giancarlo Landini, Filippo Pieralli, Francesco Corradi, Salvatore Lenti, Rino Migliacci, Carlo Nozzoli, Maddalena Grazzini, Lucia Ciucciarelli, Alessandro Morettini, Sara Bucherelli, Alessandra Petrioli, Carlotta Casati, Mario Felici, Luciano 
Ralli, Stefano Arrigucci, Laila Teghini, Giovanni Antonio Porciello, Stefano Spolveri, Daniele Baldoni, Anna Frullini, Barbara Cimolato, Gianni Lorenzini, Alessandro Pampana, Guidantonio Rinaldi, Maria Chiara Bertieri, Raffaele Laureano, Stefano Tatini, Alberto Fortini, Chiara Angotti, Valerio Verdiani, Anna Maria Romagnoli, Irene Cascinelli, Alberto Camaiti, Nicola Mumoli, Marco Cei, Stefano Giuntoli, Massimo Alessandri, Alessandro De Palma, Maurizio Manini, Veronica De Crescenzo, Michele Piacentini, Carlo Passaglia, Giancarlo Tintori, Carlo Palermo, Alba Dainelli, Roberto Andreini, Giuseppa Levantino, Plinio Fabiani, Lucia Raimondi, Massimo Di Natale, Filippo Risaliti, Rossella Nassi, Roberta Mastriforti, Roberto Cappelli, Michele Voglino, Paola Lambelet, Stefano Fascetti, Adriano Cioppi, Valentina Carli, Alessandro Tafi, Simone Meini Emilio Santoro, Claudia Rosi. Prognostic Ability of Simplified Pulmonary Embolism Severity Index (PESI) Score in Real World: A Brief Report. International Journal of Clinical and Experimental Medical Sciences. Vol. 1, No. 2, 2015, pp. 22-26.

doi: $10.11648 /$ j.ijcems.20150102.14

\begin{abstract}
Background and aim: Prognostic stratification of acute pulmonary embolism (PE) represents the cornerstone of modern management of this potentially life-threatening disease. In the latest years, a lot of clinical prognostic models have been validated. However, these are yet underused in clinical practice, especially in real world populations. The aim of our study was to test the prognostic ability of the Simplified Pulmonary Embolism Severity Index (sPESI) score in a real world population. Methods: Data records of 452 patients that were discharged for acute PE from 28 Internal Medicine wards of Tuscany (Italy) were retrospectively analysed. sPESI was calculated in the identical manner as the original study. Prognostic ability of sPESI score for predicting in-hospital all-cause and PE-related mortality was tested by using Areas under Receiver Operating Characteristics (ROC) curve (AUC). Results: $15.2 \%$ of patients were classified as sPESI score 0 , whereas $84.8 \%$ were classified as $\mathrm{SPESI} \geq 1$. All causes of in-hospital mortality were $10.95 \%$ (5.75\% PE-related $)$ in patients with sPESI score $\geq 1$ and $0 \%(0 \%$ PE-related) in sPESI score 0 . AUC for all causes of mortality was 0.694 (95\% CI: $0.650-0.736)$, whereas it was 0.702 (95\% CI: 0.657-0.743) for PE-related mortality. Conclusion: In a real world population, sPESI is a good prognosticator for all causes of in-hospital and PE-related mortality and its use should be encouraged.
\end{abstract}

Keywords: Pulmonary Embolism, Prognosis, PESI Score

\section{Introduction}

Acute pulmonary embolism (PE) remains one of the leading causes of mortality and morbidity in cardiovascular setting, especially when is associated with hemodynamic instability (1).

Prognostic stratification of acute PE is of utmost importance for the choice of appropriate treatment and setting of care. It can be assured by using clinical, instrumental and laboratory assessment $(2,3)$.

In the latest years, many prognostic models for mortality risk stratification in acute PE have been proposed. Of them, simplified PESI score (sPESI) showed to be a good prognosticator for 30-day mortality (4). The sPESI score considers the presence or absence of age over 80 years, history of cancer, heart failure or chronic obstructive pulmonary disease (COPD), heart rate $(\mathrm{HR}) \geq 110$ beats for minute, systolic blood pressure $(\mathrm{SBP}) \leq 100 \mathrm{mmHg}$, oxygen arterial saturation $\leq 90 \%$.

The contemporary absence of all variables (sPESI score 0) identifies low risk patients (30-day mortality $\leq 1 \%$ ) whereas the presence of at least one of variables (sPESI score $\geq 1$ ) identifies patients with high risk (30-day mortality around $11 \%$ ) (4). Despite, sPESI score has been used as prognostic model for identifying low risk patients candidate for home treatment in clinical trials (5-7) and much recently European Society of Cardiology (ESC) has proposed a prognostic model that is based on sPESI score as first prognostic tool for subdividing low risk patients from high risk patients needing for further prognostic assessment (8), to now sPESI score is still underused in clinical practice, especially in real world population.

Therefore, the aim of the present study was to test the prognostic ability of sPESI score as prognosticator of all causes of in-hospital and PE-related mortality.

\section{Materials and Methods}

We performed a multicenter, observational, retrospective, cohort study aimed to evaluate characteristics and clinical management of acute PE none selected patients that were admitted in internal medicine wards of Tuscany (Italy). Demographic, clinical and prognostic data of at least ten patients consecutively were discharged for acute PE between 2012 and 2013 years from each center were retrospectively provided. SPESI was calculated in the identical manner of the study validation (4).

For testing the prognostic ability of SPESI, we calculated the Areas under Receiver Operating Characteristic (ROC) curves (AUCs) for each study endpoint.

\section{Results}

Table 1 shows the general characteristics of patients.

Table 1. General characteristics of patients.

\begin{tabular}{ll}
\hline Number & $\mathbf{4 5 2}$ \\
\hline Males/Females & $39.8 \% / 60.2 \%$ \\
Mean age (years) \pm SD & $76.01 \pm 12.34$ \\
Age $\geq 80$ years & $45.3 \%$ \\
\hline
\end{tabular}




\begin{tabular}{ll}
\hline Number & $\mathbf{4 5 2}$ \\
\hline Cancer & $30.2 \%$ \\
Cardiopulmonary diseases & $22.4 \%$ \\
Heart rate $\geq 110$ beats for minute & $19.8 \%$ \\
Systolic blood pressure $\leq 100 \mathrm{mmHg}$ & $7.3 \%$ \\
Oxygen arterial saturation $\leq 90 \%$ & $27.4 \%$ \\
All cause in-hospital mortality & $9,5 \%$ \\
PE related in-hospital mortality & $5.7 \%$ \\
sPESI score distribution & \\
0Low risk & $15.2 \%$ \\
$\geq 1$ High risk & $84.8 \%$ \\
1 & $39.2 \%$ \\
2 & $27.7 \%$ \\
3 & $12.6 \%$ \\
$\geq 4$ & $5.3 \%$ \\
\hline
\end{tabular}

Legend= PE: Pulmonary embolism, sPESI: Pulmonary embolism severity index score

452 patients were enclosed in the study. $15.2 \%$ of patients were classified as SPESI score 0 , whereas $84.8 \%$ were classified as $\mathrm{SPESI} \geq 1,66.9 \%$ of patients being classified as sPESI 1 or 2.

All causes of in-hospital mortality was $10.95 \%$ in patients with sPESI score $\geq 1$ and $0 \%$ in sPESI score $0(\mathrm{p}<0.001)$. PE-related mortality was $5.75 \%$ in patients with sPESI score $\geq$ 1 and $0 \%$ in sPESI score $0(p<0.001)$. All causes of in-hospital mortality increased from $0 \%$ in patients with sPESI score 0 to $29.1 \%$ in patients with sPESI score $\geq 4$, whereas PE-related in-hospital mortality increased from $0 \%$ in patients with sPESI score 0 to $20.8 \%$ in patients with sPESI socre $\geq 4$ (Figure 1 ).

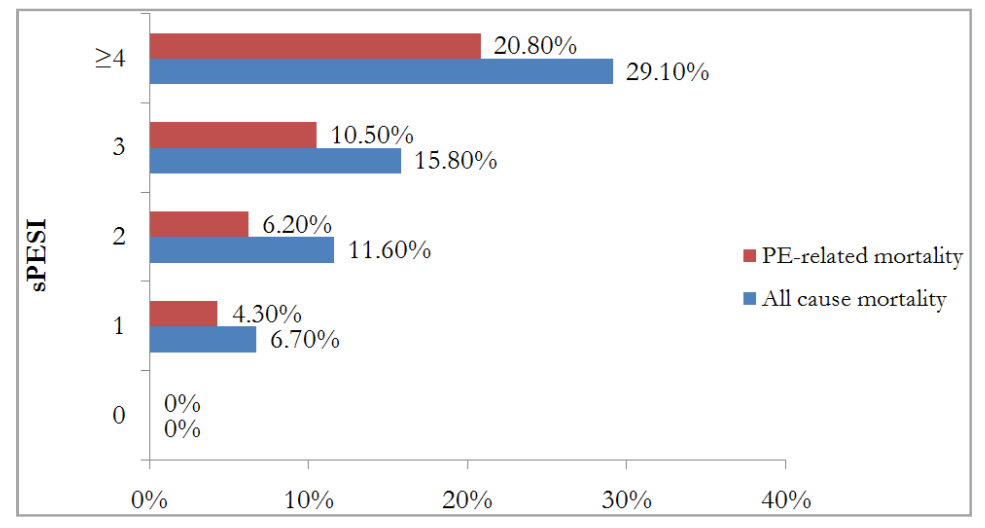

Figure 1. SPESI score and in-hospital mortality.

AUC for all causes of mortality was $0.694 \quad(95 \%$ Confidence Interval (CI): 0.650-0.736), whereas it was 0.702 (95\% Confidence Interval (CI): 0.657-0.743) for PE-related mortality. Table 2 summarizes the statistical analysis.

Table 2. Summary of statistical analysis.

\begin{tabular}{ll}
\hline $\begin{array}{l}\text { Area under the ROC curve (AUC) for all cause } \\
\text { in-hospital mortality }\end{array}$ & $\mathbf{0 , 6 9 4}$ \\
\hline Standard Error & 0,0384 \\
$95 \%$ Confidence interval & 0,650 to 0,736 \\
Z statistic & 5,055 \\
Significance level P (Area=0.5) & $<0,0001$ \\
Youden index J & 0,2674 \\
Associated criterion & $\leq 1$ \\
Sensitivity & 56,97 \\
Specificity & 69,77 \\
\hline
\end{tabular}

Criterion values and coordinates of the ROC curve [Show]

\begin{tabular}{ll}
\hline $\begin{array}{l}\text { Area under the ROC curve (AUC) for PE-related } \\
\text { in-hospital mortality }\end{array}$ & $\mathbf{0 , 7 0 2}$ \\
\hline Standard Error & 0,0507 \\
$95 \%$ Confidence interval & 0,657 to 0,743 \\
z statistic & 3,975 \\
Significance level P (Area=0.5) & 0,0001 \\
Youden index J & 0,2996 \\
Associated criterion & $\leq 2$ \\
Sensitivity & 83,80 \\
Specificity & 46,15 \\
\hline
\end{tabular}

ROC: Receiver Operating Characteristic

Figure 2 shows the ROC curves.

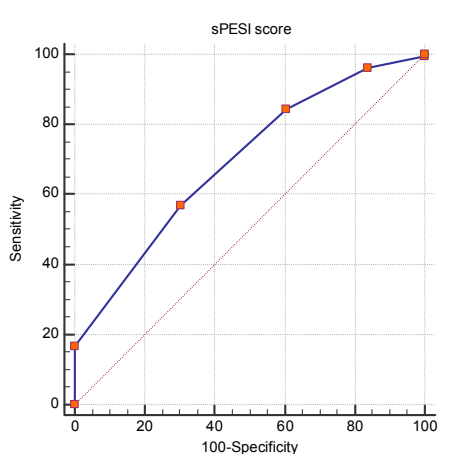

(1)

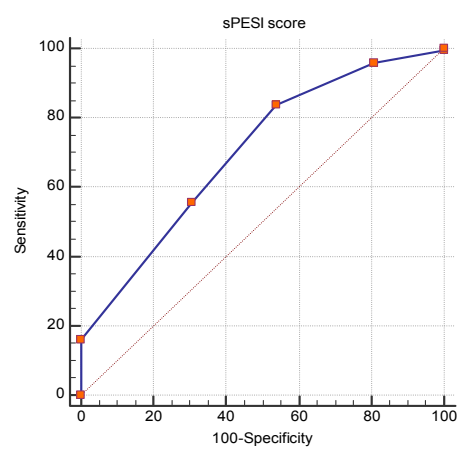

(2)

Figure 2. ROC curves for sPESI as prognosticator of in-hospital all-cause mortality (1) and PE-related mortality (2). 


\section{Discussion}

Prognostic stratification of acute pulmonary embolism (PE) represents the cornerstone of modern management of this disease (9). sPESI score is now considered as a safe prognostic model that is able to classify patients in 30-day low mortality risk (sPESI 0) and high mortality risk (sPESI $\geq 1$ ). A meta-analysis showed that the odds ratio for all causes of mortality in low risk sPESI versus high risk (3 events on 770 patients with low risk sPESI versus 72 events on 1404 patients with high risk SPESI) was 0.09 (95\% CI: 0.03-0.26), whereas it was 0.08 (95\% CI: $0.04-0.16)$ for PE-related mortality (8 events on 1003 patients with low-risk sPESI versus 205 events on 1981 patients with high risk SPESI) (10).

Prognostic stratification by using sPESI score is of utmost importance for making decision on appropriate treatment and setting of care. New ESC recommendations on PE management suggest that patients with low risk sPESI score should not receive further investigations such as right heart dysfunction and myocardial damage assessment and could be early discharged from hospital and/or treated at home after diagnostic and prognostic assessment are performed in emergency department (8). Much recently, a meta-analysis of studies on home treatment of low risk PE, a lot of them classifying low risk patients by using sPESI score, has demonstrated that 14-day and 90-day all causes of mortality were $0.41 \%$ and 1.58 , respectively (11).

From a true real world perspective, our study confirms that mortality may be extremely low if not completely absent in low risk sPESI patients.

\section{Conclusion}

Prognostic stratification is a key point in the modern management of acute PE. Despite it was limited by the retrospective methodology, our study was performed in more than $80 \%$ of internal medicine wards of one of the most populated regions of Italy, demonstrates that sPESI score, a simple and easily performable score, seems to be a good prognosticator for stratifying the in-hospital prognosis. Therefore, its wide use should be strongly encouraged.

\section{Appendix}

The Simplified Pulmonary Embolism Severity Index (sPESI)

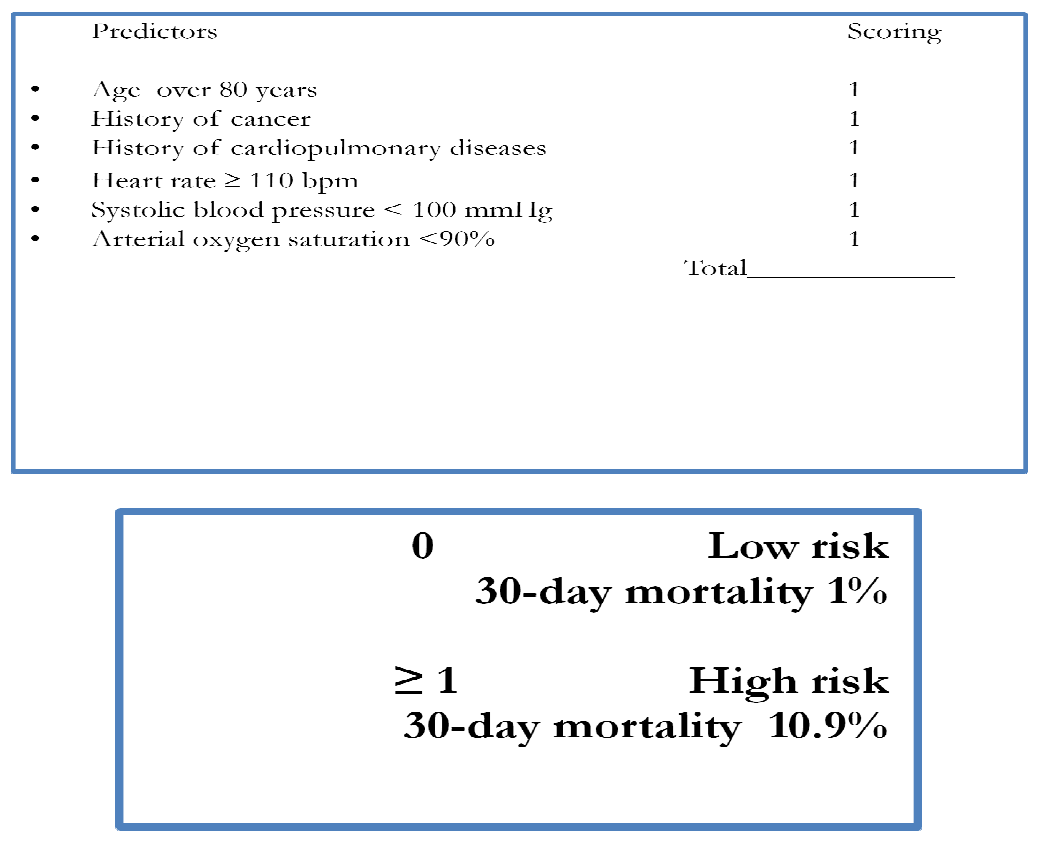

\section{References}

[1] Wood KE. Major pulmonary embolism. Review of a pathophysiologic approach to the golden hour of hemodynamically significant pulmonary embolism. Chest 2002; 121: 877-905.

[2] Masotti L, Righini M, Vuilleumier N, Antonelli F, Landini G, Cappelli R, Ray P. Prognostic stratification of acute pulmonary embolism: focus on clinical aspects, imaging, and biomarkers. Vasc Health Risk Manag 2009; 5: 567-75.

[3] Becattini C, Agnelli G. Acute pulmonary embolism: risk stratification in the emergency department. Intern Emerg Med 2007; 2: 119-29

[4] Jiménez D, Aujesky D, Moores L et al.; RIETE Investigators. Simplification of the pulmonary embolism severity index for prognostication in patients with acute symptomatic pulmonary embolism. Arch Intern Med 2010; 170: 1383-9.

[5] Lankeit M, Konstantinides S. Is it time for home treatment of pulmonary embolism? Eur Respir J 2012; 40(3): 742-9.

[6] Squizzato A, Galli M, Dentali F, Ageno W. Outpatient treatment and early discharge of symptomatic pulmonary embolism: a systematic review. Eur Respir J 2009; 33: $1148-55$. 
[7] Aujesky D, Roy PM, Verschuren F, Righini M, Osterwalder J, Egloff M, Renaud B,Verhamme P, Stone RA, Legall C, Sanchez O, Pugh NA, N'gako A, Cornuz J, Hugli O, Beer HJ, Perrier A, Fine MJ, Yealy DM. Outpatient versus inpatient treatment for patients with acute pulmonary embolism: an international, open-label, randomized, non-inferiority trial. Lancet $2011 ; 2 ; 378(9785): 41-8$.

[8] Authors/Task Force Members, Konstantinides S, Torbicki A, Agnelli G etv al.; Authors/Task Force Members. 2014 ESC Guidelines on the diagnosis and management of acute pulmonary embolism: The Task Force for the Diagnosis and Management of Acute Pulmonary Embolism of the European Society of Cardiology (ESC) Endorsed by the European Respiratory Society (ERS). Eur Heart J 2014; 35: 3033-69.
[9] Masotti L, Mannucci A, Antonelli F, Maurini V, Testa R, Marchetti S, Landini G, Cappelli R. The Risk-based Treatment of Acute Pulmonary Embolism. J Clin Med Res 2009; 1: 1-7.

[10] Zhou XY, Ben SQ, Chen HL, Ni SS. The prognostic value of pulmonary embolism severity index in acute pulmonary embolism: a meta-analysis. Respir Res 2012; 13: 111

[11] Piran S, Le Gal G, Wells PS, Gandara E, Righini M, Rodger MA, Carrier M. Outpatient treatment of symptomatic pulmonary embolism: a systematic review and meta-analysis. Thromb Res 2013; 132: 515-9. 